\title{
Valoración de la cuenca media-alta del río Cali, Colombia y su importancia para el bienestar de la fauna silvestre local
}

\author{
Assessment of medium-high basin the river Cali, Colombia and its relevance to \\ welfare local wildlife
}

GONZÁLEZ-PALOMINO, GUSTAVOํํํ.Sc, ARANGO-BUELVAS, LEÓN¹ M.Sc.

1 Universidad de Sucre, Colombia. Programa de Economía, Colombia.

\section{Keywords:}

Natural resources; econometric methods; costs of pollution control; distributional effects; green economy.
Palabras Clave:

Recursos naturales; métodos econométricos; Costos del control de la contaminación; efectos distributivos; economía ecológica.

INFORMACIÓN Recibido: 21-09-2016; Aceptado: 30-11-2016. Correspondencia autor: leon.arango@unisucre.edu.co; gustavo.gonzalez@unisucre.edu.co

\begin{abstract}
The valuation of environmental goods is a development front in applied research in economics. Colombia from travel cost method with multinomial econometric testing of orderly frequency - in this case the willingness to pay of visitors to the Cali River basin is valued. In that sense, it is taking into account the importance of local wildlife and its effect on the frequency of visits. environmental economic valuation, methodological definition, as well as a description of the relevant variables for evaluation and estimation of the basin will be addressed in this study defining the most appropriate functional form. It concludes that there is a willingness to pay for use of the inhabitants of recreational activities of US dollars 53, which infers that the basin has use and value money for its users.
\end{abstract}

\section{Resumen}

La valoración de bienes ambientales es un frente de desarrollo en investigación aplicada en economía. En este caso se valora la disponibilidad a pagar de los visitantes de la cuenca del rio Cali - Colombia a partir del método de costo del viaje con la contrastación econométrica de frecuencia multinomial ordenada. En ese sentido, se tiene en cuenta la importancia de la fauna silvestre local y su afectación por la frecuencia de las visitas. Se abordará en este estudio la valoración económica ambiental, definición metodológica, como también una descripción de las variables relevantes para valoración de la cuenca y estimación definiendo la forma funcional más adecuada. Se llega a la conclusión que existe una disponibilidad a pagar por uso de los habitantes de actividades recreativas de US 53 dólares, lo cual infiere que la cuenca presenta uso y valor monetarios para sus usuarios. 


\section{Introducción}

La creciente preocupación por la conservación de espacios naturales categorizados o no como áreas silvestres protegidas, es un asunto creciente a nivel mundial (NAREDO, 1993). Ello radica en el reconocimiento del papel que juegan estas áreas en el suministro de una serie de servicios ambientales (captura de carbono, estabilidad de ciclos hidrológicos, control de erosión, uso recreativo, valor simbólico cultural del paisaje, etc) (NAREDO, 1993). Sin embargo, su gestión resulta sumamente compleja en sociedades cuya economía es gobernada por el paradigma del mercado, debido a la dificultad de asignarles valores económicos por la ausencia de mercados reales en donde puedan ser intercambiados (FIELD, 1995).

Con el objetivo de establecer el valor que poseen para la sociedad los bienes y servicios ambientales que no tienen precio de mercado, se han desarrollado distintos métodos de análisis económico, entre ellos el de Coste de Viaje, que - igual que los métodos de Costos Evitados o Inducidos y Precios Hedónicos- infieren el valor a partir del que las personas le confieren al bien en cuestión, analizando el comportamiento de éstas en mercados de bienes con los que el recurso a valorar está relacionado (RIERA y FARRERAS, 2004).

Teniendo en cuenta que los incendios de cobertura vegetal (ICV) pueden generar profundas transformaciones en los atractivos que tienen las cuencas hidrográficas, ya que, entre otras cosas, modifican el paisaje natural al eliminar la vegetación, ahuyentan las especies de fauna silvestre motivo de interés por parte de los visitantes y alteran las condiciones particulares de los sitios utilizados para el disfrute de las fuentes de agua, se ha considerado importante adelantar un estudio que permita resolver el siguiente interrogante: ¿Es posible valorar indirectamente el impacto económico de estos eventos en el escenario de la Cuenca del Río Cali en Colombia? Por ello, en el presente trabajo se tiene como propósito valorar la Cuenca del Rio Cali a partir del método del Costo del Viaje, considerando la valoración ambiental, como también la definición de un modelo que determina la disponibilidad a pagar por parte de los visitantes de esta cuenca con contrastación econométrica de frecuencia multinomial ordenada (CABRER et al, 2001).

En este sentido, el trabajo luego de la anterior introducción, está organizado de la siguiente manera: Inicialmente se enmarca el contexto de fauna silvestre de la cuenca, como también su ubicación espacial; posteriormente una sección donde se presentan los argumentos teóricos económicos que justifican la valoración económica de la Cuenca Media - Alta del Rio Cali. En la siguiente sección se estructura lo referente a los materiales y métodos; le sigue la sección que presenta resultados de orden descriptivo y econométrico que encaminan hacia una valoración de la cuenca. Finalmente, una sección sobre la discusión de las evidencias del estudio.

\section{Descripción de la fauna del sector y ubicación espacial}

Con relación a la fauna de la cuenca, de acuerdo con CVC (2000), HERRERA (2007) y Fundación Jardín Botánico de Cali y Asociación Río Cali, citados por URCUQUI (2011) aún permanecen 186 especies de aves residentes, algunas amenazadas como el saltarín dorado Xenopipo flavicapilla y la tangará multicolor Chlorochrysa nitidissima. Adicionalmente se encuentran 12 especies de aves migratorias, entre las que sobresale la reinita alidorada Vermivora chrysoptera. Por la especialización frugívora existen especies para la conservación como el pavón Chamaepetes goudoti, la soledad de montaña Pharomacrus antisianus y el gallito de roca Rupicola peruviana.

En cuanto a los mamíferos, se destaca el yaguarandí Puma yagouaroundi, y el puma Puma concolor spp, Por otro lado, está el mico nocturno Aotus lemurinus lemurinus. Entre los grupos más abundantes de mamíferos se encuentran los murciélagos y roedores (URCUQUI, 2011).

Por otra parte, agrega que la herpetofauna cuenta con 25 especies de reptiles y 22 de anfibios. En cuanto a insectos, referenciando a RODRIGUEZ (2008) y SALGUERO (2008), URCUQUI (2011) afirma que se conocen cerca de 130 especies de mariposas diurnas y 58 de hormigas. Dentro de estas últimas se encuentran las subfamilias 
Myrmicinae, hormigas cazadoras o Ponerinae, Formicinae, Pseudomyrmecinae, Dolichoderinae, Ectatomminae y Ecitoninae.

Según el estudio de URCUQUI (2011), la cuenca está localizada al noroccidente del municipio de Santiago de Cali en lado oriental de la cordillera occidental, con un recorrido que va desde los 4050 msnm, que empieza en límite del Parque Nacional Farallones hasta el Zoológico de Cali con 1100 msnm. La cuenca esta entre las coordenadas 1.059.000 E, $847.000 \mathrm{~N}$ y $10.411 .000 \mathrm{E}, 874.000$ $\mathrm{N}$. La cuenca tiene una extensión aproximada de 12 hectáreas, donde el rio Cali con $5 \mathrm{~km}$ de longitud, está determinando un área de influencia de 34 km (URCUQUI, 2011).

\section{Referentes teóricos del estudio}

La valoración de un bien ambiental pasa por elementos subjetivos, propios de la posición del investigador, pero desde el punto de vista económico ambiental, la posición económica ambiental "acomodativa" (PEARCE y TURNER, 1990), es la alternativa de mayor referencia de los estudios del tema, los cuales tienen una condición más globalizante en los valores instrumentales incluyendo todos los valores de uso y no uso. El problema del valor de los bienes ambientales justamente se centra en que no refleja un precio (BENGOCHEA, 2003), y contrario a la postulación de la teoría del valor neoclásico, donde existe la igualdad entre precio y valor en un equilibrio. Para el caso de los bienes ambientales posiblemente la no equivalencia entre precio y valor está justificada por la no existencia de un mercado (AZQUETA, 1994).

El tema de la valoración económica ambiental se centra sobre discusiones que van en la relación entre un alto desarrollo tecnológico y el grado de devastación del medio ambiente. Por el contrario, ese alto nivel de desarrollo económico debería traer consigo mejoras en los procesos productivos que eviten el deterioro de los recursos naturales (PEARCE y TUNNER, 1990).

La aplicación de supuestos económicos ambientales, surgen en sus inicios de análisis centrados en externalidades sobre bienes de mercado, los cuales son condicionantes para una valoración ambiental. En este caso los condicionantes para valorar deben darse con estos escenarios, según AZQUETA (1994):

Una actividad de un agente provoca la pérdida o una mejora de bienestar a otro agente.

La pérdida de bienestar no está compensada o la mejora del bienestar no está explicita en una disposición a pagar. Si se propone que el tipo de bien mejora los niveles productivos de un mercado, probablemente si todos los elementos que contribuyen a la producción están constantes, menos los niveles del bien ambiental, existiría una mejora del nivel de producción y probablemente tendríamos elementos de valoración monetaria del bien ambiental, y así discriminaríamos como transarlo.

La literatura económica ambiental, presenta opciones para este tipo de valoración, pero son particulares a la especificidad de lo que quiera valorar. Se presentan 4 opciones (AZQUETA, 1994): Método de costo evitado, Método de costo del viaje, Método de precios hedónicos y Método de valoración contingente.

Para el presente trabajo se presenta una breve descripción del método del coste de viaje, dado que este método es el escogido por ésta investigación para valorar la cuenca media - alta del Rio Cali y además se adapta a los supuestos económicos en una metodología de valoración.

Método del coste de viaje: consta de la explicación de la complementariedad, partiendo de un acceso a un bien ambiental, que se debe incurrir en el consumo de bienes privados buscando lo máximo que gasta esa persona para acceder a ese bien ambiental (ARIAS, 2001; RIERA, 2000).

Este método se aplica a la valoración de áreas naturales que participan en la función de producción de utilidad de un individuo, teniendo en cuenta información sobre la utilización del bien ambiental. Asimismo, se debe tener presente el costo de acceder al bien ambiental y aquí se consideran dos tipos de costos, los ineludibles, que se refieren a los derivados del desplazamiento de la familia o persona hacia el bien ambiental y los discrecionales, son los que se incurren de una manera innecesaria y discreta mientras ocurre el desplazamiento, como comer algo, por ejemplo. 
Otro costo que se debe tener en cuenta en el coste de desplazamiento o viaje es el tiempo que transcurre durante el viaje para llegar al lugar y el tiempo pasado en dicho lugar de destino y se puede valorar como el costo de oportunidad de haber utilizado ese tiempo trabajando o efectuando otra actividad que genere utilidad (AZQUETA, 1994).

Valoración de la cuenca media - alta del Rio Cali: En el caso de valorar una cuenca como un activo o bien ambiental, se da por supuestos que permitan mostrar a un individuo la disponibilidad a pagar, dependiendo del bien ambiental y su relación con los bienes de mercado. La valoración de la cuenca parte como supuesto: la condición de disfrute de un individuo que toma la decisión de transportarse hasta el sitio. Para estos casos, en la literatura ambiental se recurre al supuesto económico de la complementariedad entre bienes que presenta el método de Costo del Viaje (AZQUETA, 1994). Por esto, el estudio recurrirá a éste método, dado las similitudes en la valoración que presenta con el método (CRITECHE y PENNA, 2008).

En el caso de la valoración de la cuenca del rio Cali, se tienen condicionantes sobre el gasto de los visitantes de la cuenca, los cuales tienen como motivo de su visita en su gran mayoría la recreación, donde el valor de disfrute del bien constituye un cambio en el bienestar consignado en la teoría de elección de bienes del consumidor (NICHOLSON, 2004), que depende del consumo de bienes. Partiendo de una función de utilidad de un consumidor, los bienes que son input, al variar positivamente, determinan a la función bienestar, aumenta positivamente, es decir una variación positiva del bienestar está justificada por el consumo de la cuenca. El punto para valorar este disfrute, presenta dificultades, ya que el cambio del bienestar es un elemento subjetivo y heterogéneo de todos los que asisten en la cuenca, adicionalmente una función de utilidad indirecta (valorando el bienestar) depende de los precios de mercado, los cuales son una incógnita para el bien ambiental. Por eso es imperante partir del supuesto de la valoración de un bien que cambia el bienestar de un consumidor, en la medida que aumente el consumo de bienes que sean complementarios al ambiental, pero que sean transados en un mercado (Tienen un precio)
(AZQUETA, 1994).

Modelo Teórico: Un método de costo del viaje tiene el supuesto que los bienes que complementan la visita a la cuenca son los determinantes a la hora valorar el bien ambiental. La primera idea en modelación del costo del viaje fue HOTELING en 1947 para valorar en términos económicos el uso recreativo de los parques en Estados Unidos, con una primera aplicación en 1966 con CLAWSON y KNETSH. Según la literatura económica ambiental, es necesario detectar los bienes que realmente sean los que complementan a la cuenca, ya que es posible escoger bienes que no sean determinantes, bienes discrecionales (AZQUETA, 1994). A pesar de las dificultades de un método de costo del viaje, los beneficios son contundentes a la hora de valorar un bien ambiental. En este caso la Cuenca del Rio Cali es fundamental para la ciudad de Santiago de Cali, como los distintos ecosistemas que lo conforman (URCUQUI, 2011).

Para el caso del Costo del Viaje, se recurre a funciones de demanda implícitas definidas por las visitas de los consumidores del bien ambiental para el disfrute de actividades recreativas $u$ ocio (AZQUETA, 1994).En esta investigación la relación funcional de las visitas con los regresores que determinan estará en la métodos y recursos. Materiales y métodos

Función económica explicita: Este trabajo se propuso modelar y valorar la cuenca media - alta del Rio Cali, y la recomendación de la literatura económica es seguir la modelación propuesta por AZQUETA (1994), CRISTECHE y PENNA (2008) y las distintas perspectivas de modelos de demanda que muestra RIERA (2000), por tanto, se propone explicar el número de visitas mensuales por variables económicas, las cuales muestran una relación directa e indirecta al número de visitas. Se parte como los costos asociados por visita afectan indirectamente al número de visitas y la totalidad de estas a la fauna silvestre. En este caso las variables explicatorias para el número de visitas son:

Económicas: Costo monetario de emplazamiento e Ingreso.

Sociales: Género, nivel de escolaridad y edad. Percepción de satisfacción: Grado de satisfacción de la visita, Atributos del sector, 
Tiempo de la visita y cantidad de visitantes que acompañan en la visita.

Disponibilidad a pagar: pago por la visita y aporte de trabajo voluntario.

Por tanto, el número de visitas estaría explicada por:

$V_{i}=f\left(C_{i}, Y_{i}, G_{i}, E_{i}, E d_{i}, S_{i}, t_{i}, V i s_{i}, D P_{i}, T r_{i}, \varepsilon_{i}\right)$

$V_{i}=f\left(C_{i}, Y_{i}, G_{i}, E_{i}, E d_{i}, S_{i}, t_{i}, V i s_{i}, D P_{i}, \operatorname{Tr}_{i}, \varepsilon_{i}\right)$

(1)

Dónde:

Variables continúas

$\mathrm{Vi}=$ Número de visitas de frecuencia mensual

$\mathrm{Ci}=$ Costo total de emplazamiento, medido en pesos

Yi = Pago de servicios públicos (Variable proxy de ingreso), medido en pesos

$\mathrm{Ei}=$ Años de escolaridad, como último año cursado

$\mathrm{Edi}=\mathrm{Edad}$ del encuestado

$\mathrm{t} i=$ Tiempo de la visita en horas

Vis = Cantidad de acompañantes de la visita

Dpi = Disponibilidad a pagar por visitar la cuenca, medido en pesos

Tri $=$ Horas de trabajo como trabajo voluntario

\section{Variables categóricas}

$\mathrm{Gi}=1$, si el individuo es hombre

$\mathrm{Si}=1$, si el individuo manifiesta una alta o completa satisfacción por la visita.

\section{Variables Estocásticas}

ei $=$ Termino de perturbación aleatoria

En este caso, se presenta el conjunto de variables explicatorias al número de visitas mensuales a la cuenca del rio Cali, tal como está planteado en el modelo referenciado por AZQUETA (1994), de demanda individual de un bien ambiental y aplicado en TOBIAS y MENDELSOHN (1991) [Tomado de IZKO, BURNEO. (2003)] en condiciones de función de demandas de recreación.

Las variables explicatorias, presentan una lógica económica para afectar a la cantidad de visitas mensuales. En este sentido, los costos de emplazamiento si aumentan disminuyen el nivel de visitas al bien ambiental. El nivel de ingreso representado por el nivel de pago de servicios públicos (Supuesto de amplia referencia en modelos de consumo, donde los encuestados difícilmente revelan sus ingresos), muestra una relación directa con la frecuencia de visitas mensuales (GONZALEZ, 2000).
Frente a los regresores o variables explicativas de referencia social, se tiene al género, el cual mostraría una mayor frecuencia de visitas si el individuo es hombre. Como también un mayor nivel de escolaridad debería aumentar el nivel de vistas mensuales al bien ambiental. Respecto a la edad, el efecto puede ser similar al nivel de escolaridad (GONZALEZ, 2000).

Las variables de percepción de satisfacción muestran una relación directa con la frecuencia de visitas al bien ambiental. El tiempo de la visita puede tener resultados ambiguos, si se presentan visitas cortas, pero de mayor frecuencia mensual, pero podría tener el mismo efecto para visitas largas, que representan un domicilio para el individuo. La cantidad de acompañantes a la visita del bien ambiental deberá tener una relación directa con la frecuencia mensual (GONZALEZ, 2000).

La disponibilidad a pagar, mostraría una alta valoración del bien ambiental, el cual está reflejado en una alta frecuencia de visitas, dándose una relación directa, bien sea con un aporte pecuniario o tiempo de voluntariado en preservación del activo ambiental (FIELD, 1995).

\section{Puntos de recolección de la información} primaria: Para poder modelar la frecuencia de visitas a la cuenca del rio Cali, a partir de los regresores anteriormente mencionados, la información primaria es generada utilizando como instrumento una encuesta, la cual revelaría la variabilidad de aquellos, como también de la variable a explicar. Dentro de las pruebas piloto se encontró 4 áreas de mayor frecuencia de visita a la cuenca, en este caso son:

Río Cali, sector del Jardín Botánico, el cual es altamente concurrido especialmente por habitantes de la Comuna uno y de otros sectores populares de la Ciudad de Cali.

Sector de Venteaderos, ubicado en la vía CaliPichindé. Se caracteriza por ser un sector confluencia de vías (Venteaderos-Pichindé y Venteaderos-Yanaconas), y senderos y de sitios con distintos atributos (baño: ríos Pichinde y Pichindecito; religiosos: Monumento a la virgen de Yanaconas; etc.).

Centro Recreacional de Yanaconas, establecimiento cerrado con un alto volumen de visita perteneciente a la Caja de Compensación 
COMFENALCO. Además de las áreas internas de recreación, dispone de senderos y otros espacios para el desarrollo de actividades a campo abierto. Monumento de Cristo Rey, constituye el principal mirador de la Ciudad, cuenta con un alto volumen de visita los fines de semana y días festivos.

Como el método de valoración del costo del viaje es indirecto, en cuanto el bien ambiental a valorar no presenta un precio de transacción, se recurre a un supuesto de complementariedad del bien ambiental con los bienes de mercado consumidos en la visita; el instrumento de generación de la información refleja esta valoración indirecta de la cuenca del rio Cali.

Definición del espacio muestral: Para encontrar la cantidad de encuestados de la ciudad de Cali, es prioritario definir el universo a partir de los beneficiarios del emplazamiento (HERRADOR y DIMAS, (2001). Para el caso del estudio, la GEIH (Gran Encuesta Integrada de Hogares, reportada por el DANE) del año 2007 en el mes de junio reporta una frecuencia mensual de 800 hogares en el área metropolitana de Cali - Yumbo. En este caso, el muestreo a partir de la representación del número de hogares de la $\mathrm{ECH}$, revelaría una población de 700000 familias, donde un muestreo en el estudio de valoración considera una población finita:

$n=\frac{N * Z_{\alpha}^{2} * p * q}{d^{2} *(N-1)+Z_{\alpha}^{2} * p * q}=268$

Dónde:

$\mathrm{N}=$ Total de la población $=700000$

$Z_{a}{ }^{2}=1.65^{2}$ (si la seguridad es del $90 \%$ )

$p=$ proporción esperada (en este caso $50 \%=0,5$ )

$q=1-p($ en este caso $1-0.5=0,5)$

$d=$ precisión (en este caso deseamos un $5 \%$ ).

Para el caso de estudio se tiene un nivel de significancia del $90 \%$ y margen de error del $5 \%$, que muestran 268 encuestados.

\section{Resultados}

Caracterización de la información de la muestra: En la Tabla 1, se presenta información sobre las variables continuas, producto de la muestra del estudio:
Tabla 1. Descripción de las variables continúas.

\begin{tabular}{cccccc}
\hline Variable & Observaciones & Media & D.S. & Mínimo & Máximo \\
\hline Tiempo vista & 261 & 6,136015 & 9,372714 & 0 & 96 \\
Acompañantes & 255 & 4,666667 & 7,794271 & 0 & 100 \\
Costo & 233 & 27457,94 & 45652,29 & 1000 & 370000 \\
Disponib. a pagar & 20 & 56500 & 132463,8 & 500 & 600000 \\
Horas Voluntario & 119 & 2,621849 & 2,062546 & 1 & 10 \\
Pago energía & 251 & 81884,46 & 62256,68 & 10000 & 450000 \\
Edad & 251 & 36,59761 & 10,56984 & 15 & 71 \\
Nivel de escolaridad & 253 & 12,31621 & 3,308661 & 3 & 21 \\
\hline
\end{tabular}

Fuente: Encuesta realizada y cálculos propios

En este caso la baja frecuencia de disponibilidad a pagar, no muestra peso en la frecuencia de visitas. De acuerdo al coeficiente de variación de estas variables continuas, las horas de voluntariado, como el pago de energía, la edad y el nivel de escolaridad es menor a 1 , por lo que muestra cierta homogeneidad en sus respuestas, pero sigue siendo alto para horas de voluntariado, pago de energía. Siendo más homogénea la muestra para edad y escolaridad.

La referencia de estimación econométrica de un método de costo del viaje generalmente está con estimaciones mínimo cuadráticas, para que la variable dependiente (número de visitas) tenga un alto nivel de variabilidad. Para el caso, el nivel máximo de variación es 5 visitas mensuales, limitando el nivel de cambio de la variable a explicar. Si se parte de una estimación mínimo cuadrática se tendrá la predicción de una variable lineal, donde los niveles de pronóstico estarán por encima de 5 y por debajo de 1 , lo cual no se ajusta a la información de los encuestados. En el estudio RIERA (2000), se han realizado estimaciones multinomiales de máxima verosimilitud, por esto es importante plantear y tener especificaciones alternativas que reflejen condiciones propias en el estudio, por esto es importante plantear que la variable dependiente presenta una variación categórica múltiple de una forma ordenada, teniendo una referencia de estimación multinomial ordenada. En este caso se referencia el modelo Logit Ordenado (CABRER et al, 2001), donde la variable dependiente es el número de visitas mensuales a la cuenca. Se mantienen los regresores propuestos en la fundamentación teórica del modelo, pero en este 
caso, con la capacidad de modelar la probabilidad del número de visitas mensuales, con un modelo de estimación no lineal.

Modelo econométrico y sus resultados: EI modelo de respuesta múltiple ordenado relaciona la variable dependiente con los siguientes regresores, X1, X2...Xk, a través de la siguiente ecuación:

$Y_{i}^{*}=F\left(X_{i} \beta\right)+u_{i}$

Siendo $Y^{*}=$ una variable latente (no observada) que cuantifica las categorías ordenadas

$F()=$ Una función no lineal logística

$\mathrm{XiB}=$ Combinación lineal de regresores $\mathrm{y}$

parámetros

$\mathrm{Ui}=$ Ruido blanco.

Para las categorías de la variable dependiente:

1, si $Y i^{*} \leq \mathrm{c} 1$

2, si $c 1 \leq Y i^{*} \leq c 2$

$Y i=3$, si $c 2 \leq Y i^{*} \leq c 3$

4 , si $c 3 \leq Y i^{*} \leq c 4$

5 , si $c 4 \leq Y i^{*}$

Siendo ci, los puntos de corte de la variable latente, que corresponde a una función logística acumulada.

Presentación de los resultados:

$$
\begin{gathered}
\tilde{L_{i}=-0.467 * \text { Amenazas }}+0.0613 * V i s_{j}+0.00000187 * C_{i}+0.0000121 * \operatorname{Tr}_{i} \\
+0.5111 * g_{i}+0.00000858 * Y_{i}-1.18 * D 1_{i}+2.6 * D 2_{i}
\end{gathered}
$$

Ee $(0,33)$

$(0,0000024)$

$(0,318028)$

$(0,40)$

$(0,000002)$

$\mathrm{n}=216 \quad$ Test de Wald $=63,78$

$\mathrm{P}($ valor $)=0,0 \quad$ PseudoR2 $=0,19$

Puntos de Corte:

C1 (un viaje) $=2,597145$

C2 $($ dos viajes $)=3,685028$

C3 (tres viajes) $=3,725717$

C4 (cuatro viajes) $=7,117949$

Dónde:

$\mathrm{Li}=$ Variable latente

$\mathrm{Ci}=$ Costo total de emplazamiento, medido en pesos

Yi = Pago de servicios públicos (Variable proxy de ingreso), medido en pesos ti $=$ Tiempo de la visita en horas

Vis = Cantidad de acompañantes de la visita

Tri $=$ Horas de trabajo como trabajo voluntario

\section{Variables categóricas}

$\mathrm{Gi}=1$, si el individuo es hombre

$\mathrm{D} 1=1$, si visita a Yanaconas

$\mathrm{D} 2=1$, si visita al rio Cali

Amenazas $=1$, si el encuestado manifiesta el peligro a la cuenca de los incendios

El modelo trata explicar la cantidad de visitas mensual que realiza un individuo a la cuenca del rio Cali, la tendencia de la información consignada muestra un reporte de visitas mensuales a la cuenca. Para esto es importante definir si este reporte tiene una especificación lineal o si más bien estructuras basadas en las funciones de probabilidad acumulada. Para este caso la especificación de una función logística acumulada, muestra mayores resultados en bondad del ajuste y especificación en los regresores (en este caso, son los de mayor significancia estadística), por esto se utiliza una estimación de un modelo Logit ordenado. El modelo estimado muestra significancia global a partir del test de Wald, lo cual indica una buena especificación de variables explicativas escogidas; por otro lado, a nivel individual las variables significativas son la cantidad de acompañantes cuando visita la cuenca, el valor de las horas de voluntarias para la preservación de la cuenca, la proxy del ingreso (pago energía) y el efecto binario de visitar a Yanaconas como también al Rio Cali. Como la variable dependiente es una variable latente que representa el valor de una distribución de una logística acumulada, los puntos de corte muestran como la distribución del pronóstico de visitas está distribuida en una visita mensual, ya que un valor de 2,59 (c1) refleja una probabilidad acumulada mayor del $95 \%$ que los individuos de la muestra.

Respecto al valor de los estimadores se incluye al modelo original la variable amenazas de incendios (Tal como lo plantea RIERA, (2000), como atributos), el cual tiene un valor binario si considera el encuestado que los incendios son una amenaza a la cuenca, cuyo valor negativo de las amenazas de los incendios (p4) refleja que estos disminuyen la cantidad de visitas a la cuenca. Por otro lado, la cantidad de personas con 
la que visita la cuenca (vis=p7), su valor positivo muestra que a mayor cantidad de personas que visita la cuenca mayor es la cantidad de vistas mensuales. El estimador de la cantidad de dinero utilizado para las visitas al ser positivo muestra ser consistente (C), pero no es significativo. La variable género $(\mathrm{g})$ evidencia que los hombres aumentan la probabilidad de tener más visitas a la cuenca, con una significancia cercana al $10 \%$. Una variable significativa y consistente es el efecto ingreso medido en el pago de servicios públicos $(\mathrm{Y})$, ya que a mayor ingreso mayor es la probabilidad de tener visitas a la cuenca. El efecto binario de visitar el rio Cali (D2), muestra que aumenta la cantidad de visitas a la cuenca, por el contrario, el efecto de ir a Yanaconas (D1) muestra una disminución de la probabilidad de ir a la cuenca del rio Cali. Por último, se encuentra la variable horas de voluntariado al ser su estimador significativo y positivo, aumentaría la frecuencia de viajes de la cuenca.

La estimación al ser de corte transversal, generalmente es heterocedastica por eso se calcula con estimadores de varianza robusta a partir del supuesto de White (GUJARATI, 1997), donde estas varianzas están corregidas, ya que una estimación sin esta corrección determinaría un sesgo en la estimación de estas mismas.

Por el lado del pronóstico el modelo arroja que el valor más probable en todas las frecuencias en una visita. Adicionalmente una valoración de la cuenca del Rio Cali, a partir del supuesto de la complementariedad de los bienes finales consumidos para consumir la cuenca, referencia a la metodología de Costo de Viaje, donde la cuenca se define como un bien ambiental, el cual su valor económico es el que se define como los consumos de bienes finales de los fueron necesarios para llegar a él. En este caso se definió el trabajo voluntario para presérvalo (Valorado como valor de salario mínimo) y el costo de consumo de bienes para llegar a la cuenca.

Con los valores estimados, el valor promedio mensual partiendo de las disponibilidades a pagar bajo el método de estimación de costo del viaje con la técnica de Turnbull y Kriström [HERRADOR y DIMAS. (2001)] de las estimaciones es $\$ 11,120$ (US) para 211 encuestados. Per cápita la disponibilidad a pagar es $\$ 103,216.11$. (US).

\section{Discusión}

Si bien existen diversos factores que afectan un ecosistema, en el caso de la cuenca del río Cali se destaca, como amenaza al paisaje y variable que atenta contra la fauna silvestre, en primera instancia la invasion de territorio y el proceso de urbanización, a lo que se le suma los incendios forestales. Lo anterior puede ser más gravoso si aumenta el número de visitantes y el tiempo de visita al lugar señalado, toda vez que gran parte de dicha fauna está en vía de extinción.

Cabe anotar que por las acciones del hombre en su busqueda de bienestar se pueden presentar externalidades negativas y el paisaje de la cuenca del río Cali no es la excepción, de ahí que en términos de costos los visitantes los asumen no en forma monetaria directamente, sino a traves de acciones conservacionistas del paisaje, es decir en forma de trabajo voluntario.

\section{Referencias}

ARIAS, C. 2001. Estimación del valor del regadío a partir del precio de la tierra. Economía Agraria y Recursos Naturales 1:115-123

AZQUETA, D. 1994. Valoración económica de la calidad ambiental. Ed. McGraw Hill. Madrid BENGOCHEA, A. 2003. Valoración del uso recreativo de un espacio natural. Estudios de economía aplicada. Universitat Jaume 1. 21 (2):321-338.

CABRER, B.; SANCHO, A.; SERRANO, G. 2001 Microeconometría y decisión. Editorial Pirámide. Madrid.

CRISTECHE, E.; PENNA, J. A. 2008. Métodos de valoración económica de los servicios ambientales. Estudios socioeconómicos de la sustentabilidad de los sistemas de producción y recursos naturales. Instituto de Economía y Sociología (IES). Buenos Aíres, Argentina. 
CLAWSON, M.; KNESTCH, J. L. 1966. Economics of outdoor recreation. Johns Hopkins University Press for Resources for the Future. Washington.

IZKO, X.; BURNEO, D. 2003. Herramientas para la valoración y manejo forestal sostenible de los bosques Sudamericanos. Programa de Conservación de Bosques Oficina Regional para América del Sur. UICN-Sur.

FIELD BARRY. 1995. Economía ambiental, una introducción. Editorial McGraw - Hill. Bogota D.C. GONZÁLEZ, M. 2000. Preferencias de los individuos por los espacios recreativos: dos aplicaciones en Galicia. Estudios de Economía Aplicada 16:93-110.

GUJARATI. D. 1997. Econometría. Editorial McGraw Hill. Cuarta Edición. México.

HERRADOR, D.; DIMAS, L. 2001. Valoración Económica del Agua para el Área Metropolitana de San Salvador. Fundación PRISMA. El Salvador.

NAREDO, P. 1993. Hacia una ciencia de los recursos naturales. Editorial Siglo XXI. Madrid

NICHOLSON, W. 2004. Teoría microeconómica. Editorial Thomson. Octava edición. México D.F.

PEARCE, D.; TURNER.K. 1990. La economía de los recursos naturales. Editorial Celeste. Madrid

PINDYCK, R. 2001. Econometría, modelos y pronósticos. Editorial McGraw Hill. Cuarta edición. México D.F.

RIERA FONT. A. 2000. Valoración económica de los atributos ambientales mediante la metodología del costo del viaje. Estudios de economía aplicada 14: 173-198.

URCUQUI, A. 2011. Conservación y conflictos socioambientales en la cuenca Media - Alta del río Cali, Valle del Cauca. Trabajo de maestría en Desarrollo rural, Pontificia Universidad Javeriana. Bogotá. 